\title{
Aplicación De Un Modelo Basado En La Gestión Del Conocimiento Para Mejorar EI Nivel De Aprovechamiento De Las Tic. Estudio De Caso En Una Pyme Mexicana
}

\begin{abstract}
Francisco Javier León-Moreno
Estudiante de Doctorado en Planeación Estratégica

y Dirección de Tecnología

Universidad Popular Autónoma del Estado de Puebla (UPAEP). México.

Jesús Martín Cadena-Badilla

Ramón Arturo Vega-Robles

Doctor en Planeación Estratégica y Dirección de Tecnología por la UPAEP.

Maestro-Investigador. UNISON, México

Doi: 10.19044/esj.2018.v14n10p270 URL:http://dx.doi.org/10.19044/esj.2018.v14n10p270

Abstract

A very dynamic environment and constant technological advances are surrounding small and medium-sized enterprises (SMEs) in their commercial activities. The business context is becoming increasingly complex, as there are continually numerous aspects related to the application of knowledge, both financial, legal, organizacional and technological. In relation to the application of information and communication technologies (ICT), SMEs need to acquire and apply knowledge correctly to obtain the best use of these technologies in their business processes. Taking this scenario into account, the objective of this paper is to present the case study on the application of a conceptual model based on knowledge management (KM) to improve the level of use of ICT in the business processes of mexican SMEs. The model was applied in an SME of the commercial sector. The results obtained are exposed, as well as the conclusions and future perspectives of the work developed.
\end{abstract}

Keywords: SMEs, ICT, Knowledge Management

\section{Resumen}

Un ambiente muy dinámico y avances tecnológicos constantes están rodeando a las pequeñas y medianas empresas (PYMES) en sus actividades comerciales. El contexto empresarial se torna cada vez más complejo, ya que continuamente se requieren numerosos aspectos relacionados con la 
aplicación de conocimiento, tanto financiero, legal, organizacional y tecnológico. En relación con la aplicación de las tecnologías de la información y comunicación (TIC), las PYMES necesitan adquirir y aplicar acertadamente el conocimiento para obtener el mayor aprovechamiento de estas tecnologías en sus procesos de negocio. Tomando en cuenta este escenario, el objetivo de este trabajo es presentar el estudio de caso sobre la aplicación de un modelo conceptual basado en la gestión del conocimiento (GC) para mejorar el nivel de aprovechamiento de las TIC en los procesos de negocio de las PYMES mexicanas. El modelo se aplicó en una PYME del sector comercial. Se exponen los resultados obtenidos, así como las conclusiones y las futuras perspectivas del trabajo desarrollado.

Palabras Clave: PYMES, TIC, Gestión del conocimiento

\section{Introducción}

Las pequeñas y medianas empresas (PYMES) representan un papel importante en el contexto socioeconómico por su aportación a la generación de empleos y por sus contribuciones a la economía nacional (Alderete, 2013), por lo que es relevante conocer y abordar su problemática la cual suele ser muy diversa. En lo referente al uso práctico de las tecnologías de información y comunicación (TIC), la mayoría de los estudios suelen enfocarse en experiencias de las grandes organizaciones, donde la alineación estratégica es un factor clave en relación al éxito de la inversión y el provecho obtenido de las TIC. En cambio, debido a que las PYMES funcionan normalmente sin especialistas dedicados en tecnologías de información, esto propicia que no se obtenga el máximo potencial de estas tecnologías (Wilkin, 2012).

En la emergente economía basada en el conocimiento, la gestión del conocimiento (GC) se está difundiendo rápidamente en los círculos académicos y en el mundo de los negocios. La gestión eficaz del conocimiento es vital para la supervivencia y la prosperidad de una empresa (Park et al., 2013). La GC brinda un enfoque amplio y multidimensional, cubriendo la mayoría de los aspectos de las actividades de la empresa. La experiencia demuestra que para ser competitivo y tener éxito, las empresas deben crear y mantener un capital intelectual equilibrado (Wiig, 1997). Para muchas empresas, esto implica la necesidad de afinar y optimizar sus procesos de negocio (Niedermann, 2015). La capacidad de adaptar continuamente sus procesos de negocio es una habilidad crucial para que las empresas logren sobrevivir en el dinámico mundo empresarial actual. (Radeschütz et al., 2015).

Considerando el escenario anterior donde se involucran los procesos de negocio, la GC y la aplicación de las TIC en las PYMES, el propósito de este trabajo es presentar un caso de estudio de la aplicación de un modelo conceptual basado en la GC para mejorar el nivel de aprovechamiento de las 
TIC en los procesos de negocio de las PYMES en el contexto mexicano. La aplicación del modelo se realiza en una PYME del sector comercio del ramo ferretero. La estructura de este trabajo contempla un marco de referencia donde se presentan conceptos importantes relacionados con este estudio. En la siguiente sección se presenta la metodología realizada explicando cada etapa en la aplicación del modelo propuesto. A continuación se presentan los resultados obtenidos, y finalmente se presentan las conclusiones y las futuras perspectivas del trabajo desarrollado.

En comparación con las grandes corporaciones, la mayoría de las PYMES perciben las barreras de implementación de TIC en sus operaciones comerciales como una iniciativa costosa, con riesgo y un procedimiento complejo (Chong, Chan \& Ooi, 2012). Por tal razón, muchas PYMES debido a la falta de recursos internos, incluyendo la falta de conocimientos y habilidades en sistemas de información, recurren a consultores externos para obtener ayuda en los procesos de fortalecimiento tecnológico (Bradshaw, Cragg \& Pulakaman, 2012). Sin embargo, es importante considerar que muchas organizaciones fracasan en las inversiones en TIC, lo cual es especialmente cierto en las PYMES, ya que la gestión de las TIC se basa a menudo en una visión de corto plazo y prácticas informales en la toma de decisiones (Rantapuska \& Sore, 2011). Debido a esto, se vuelve un factor importante para la gestión de TIC tener en cuenta su eficacia operativa, así como promover su aplicación estratégica para apoyar los procesos de negocio internos (contabilidad, inventarios, compras, ventas, recursos humanos), y los externos (proveedores, clientes, gobierno, socios, etc.) alineando la aplicación de las TIC con la estrategia del negocio con el propósito de maximizar sus beneficios (Pedraza, Guerrero \& Lavín, 2011), ya que los activos basados en el conocimiento crean valor, haciendo de la gestión del conocimiento también una fuente de ventaja competitiva (Fey \& Furu, 2008; Wang \& Noe, 2010).

\section{Las TIC y la GC en el entorno empresarial}

Existen algunos elementos que influyen en la capacidad de adopción de TIC como son la estructura organizativa, la actitud hacia las TIC y la familiaridad con las mismas por parte del propietario o del gerente general de la empresa, que afectan fuertemente las posibilidades de incorporar estas tecnologías. (Scupola, 2009; Huaroto, 2012). De acuerdo a la complejidad y al campo de utilización de las TIC, los efectos sobre el desempeño en las empresas serán muy distintos, ya que se pueden clasificar las aplicaciones según las áreas de trabajo donde se utilicen, puede ser en áreas aprovechables para la empresa en su conjunto como es el caso del uso de sistemas de información ERP (por sus siglas en inglés "Enterprise Resources Planning" o "Planificación de Recursos Empresariales") e Intranet, así como también orientadas a las relaciones entre empresas como como son el uso de Extranet 
(Miyazaki, Idota \& Miyoshy, 2012). Por otro lado, se tiene que la presión competitiva y la relación con clientes y proveedores pueden influenciar positivamente la intención hacia la incorporación y utilización de TIC en las empresas (Chinedu \& Chen, 2014).

En el contexto empresarial, la GC y la innovación se consideran estrategias importantes para mejorar la capacidad de una organización para responder a los requisitos cambiantes de la tecnología y poder mantener un desempeño competitivo en el entorno empresarial (Al-Sa'di et al., 2017). La aplicación efectiva del conocimiento se manifiesta entonces en nuevas formas de valor para la organización, incluyendo nuevas ideas, productos y procesos. (Dahiyat, 2015). En la literatura se ha encontrado algunos factores que han impedido que las PYME adopten prácticas de GC, como son la escasez de recursos tanto humanos como financieros. Por otra parte, las TIC están disminuyendo el peso de estos factores, reduciendo las barreras humanas y financieras que impiden su adopción (Cerchione et al., 2015).

\section{Metodologia}

La metodología consiste en aplicar el modelo propuesto (ver figura 1), siguiendo cada una de las etapas que lo conforman, con el fin de mejorar el nivel de aprovechamiento de las TIC en los procesos de negocio. El modelo se aplicó en una PYME ubicada en el noroeste del estado de Sonora, México. Esta empresa se dedica a la compra-venta de productos del ramo ferretero, cuenta con 30 empleados, los cuales realizan ciertas actividades relacionadas con los procesos básicos del negocio como son ventas, compras, cuentas por cobrar, cuentas por pagar y control del inventario. Como apoyo a estos procesos se utiliza un sistema de información ERP comercial, que se encuentra instalado en red para el procesamiento de la información. Cabe destacar que el sistema ERP es de código semi-abierto, lo cual permite realizar adecuaciones personalizables a los procesos requeridos por la empresa. Se contactó a programadores certificados que tuvieran los conocimientos y la experiencia que permitieran implementar soluciones para resolver las necesidades planteadas por la empresa. Las respuestas a las preguntas en la tabla 1 se tomaron como base para seleccionar al proveedor de TIC. 


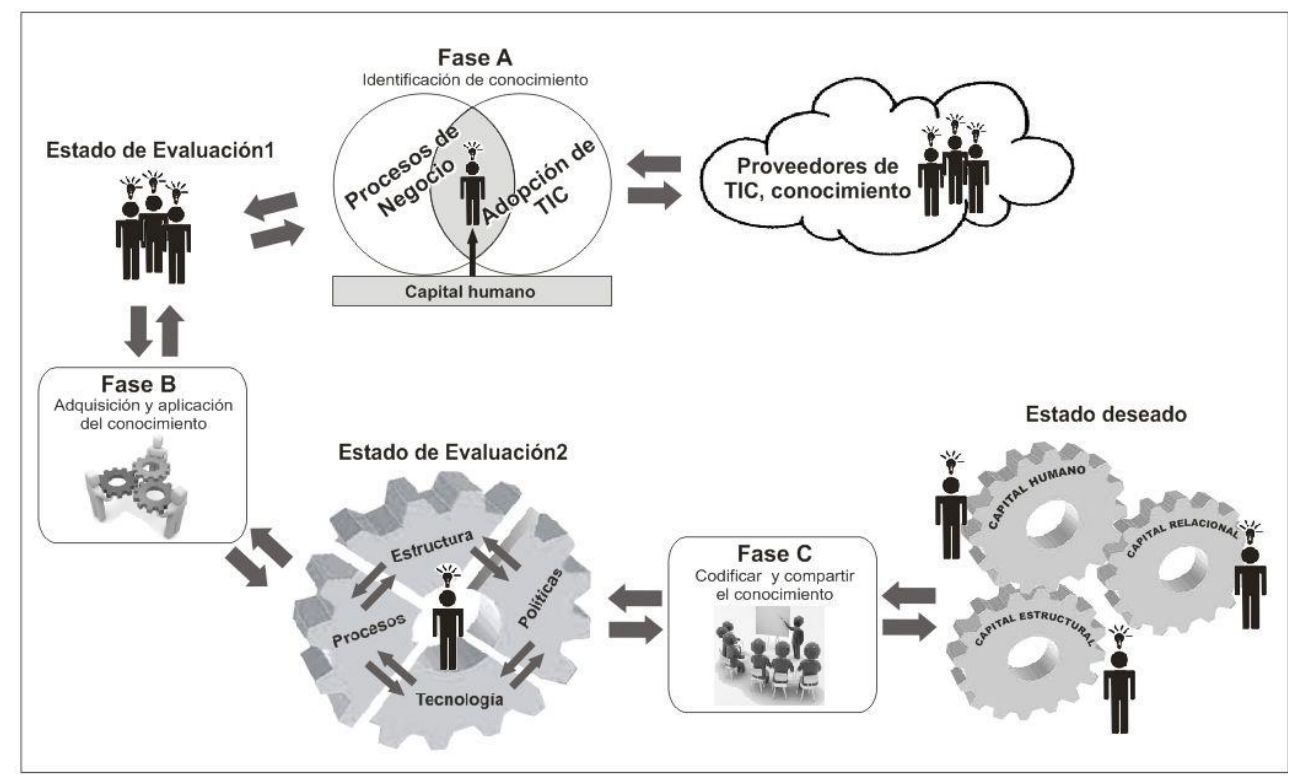

Figura 1.- Modelo conceptual propuesto basado en la GC para mejorar el nivel de aprovechamiento de las TIC.

\section{Fase A: Identificación del conocimiento.}

Esta fase consiste en determinar el estado actual, identificar el conocimiento existente y las necesidades de conocimiento de la PYME en relación a las TIC y sus procesos, así como su relación con proveedores externos que estén en condiciones de asesorar a la empresa. Para ello se plantearon preguntas relacionadas con los proveedores, tecnología, procesos, capacitación, recursos humanos, costos, tiempo de implementación, así como al cumplimiento de objetivos tomando en cuenta las preguntas relacionadas con ¿Quién? ¿Cuál? ¿Dónde? ¿Cómo? ¿Por qué? ¿Cuándo? ¿Cuánto?

El proceso seleccionado para la aplicación del modelo fue el proceso de compras de productos. Este proceso es de vital importancia, ya que es por medio del cual se ponen a disposición los productos que la empresa debe vender para satisfacer las necesidades de sus clientes. En la tabla 1 se muestra una serie de preguntas en relación con el grado de conocimiento sobre el proceso de compras y la aplicación de las TIC. Estas preguntas fueron planteadas por el gerente general, el asistente del gerente, el encargado de compras y el auxiliar del almacén. 
Tabla 1.- Algunas preguntas relacionadas con el proceso de compras y la aplicación de las TIC.

\begin{tabular}{|c|c|}
\hline \multicolumn{2}{|c|}{ Análisis previo del proceso de compras en relación con la aplicación de las TIC } \\
\hline $\begin{array}{c}\text { Proceso de negocio } \\
\text { Nombre del proceso: COMPRAS }\end{array}$ & $\begin{array}{c}\text { Aplicación de TIC } \\
\text { (Evaluación del perfil del proveedor } \\
\text { TIC) } \\
\end{array}$ \\
\hline $\begin{array}{c}\text { ¿Cuál es el propósito de este proceso? } \\
\text { ¿Cuáles son las actividades involucradas? } \\
\text { ¿Quién realiza el proceso? } \\
\text { ¿Cuándo se realiza el proceso? } \\
\text { ¿Cuánto tiempo toma el proceso y como se } \\
\text { realiza? } \\
\text { ¿Con que frecuencia se realiza el proceso? } \\
\text { ¿Cuáles otras áreas se relacionan con el } \\
\text { proceso? } \\
\text { ¿Cómo se mejorará el aprovechamiento de } \\
\text { las TIC en este proceso? }\end{array}$ & $\begin{array}{c}\text { ¿Se tiene conocimiento de cómo aplicar las } \\
\text { TIC en este proceso? } \\
\text { ¿Se ha identificado quienes pueden brindar } \\
\text { la tecnología y el conocimiento adecuado } \\
\text { para aplicarse al proceso? } \\
\text { ¿Qué ventajas se obtendrían con la } \\
\text { aplicación de las TIC? } \\
\text { ¿Cuál es el presupuesto requerido y cuánto } \\
\text { tiempo toma la implementación de TIC? } \\
\text { ¿Se cuenta con personal para el uso de las } \\
\text { TIC en este proceso? }\end{array}$ \\
\hline
\end{tabular}

Fuente: Elaboración propia.

\section{Estado de evaluación 1}

Consiste en dar respuesta y evaluar todos los puntos establecidos en la fase A. En este caso el personal de la empresa involucrado en el proceso de compra brindó las respuestas a las preguntas relacionadas con este proceso. Del mismo modo se hicieron las preguntas y se aplicó la evaluación a tres posibles proveedores, de los cuales uno de ellos mostró un perfil adecuado en relación a los conocimientos necesarios para implementar las TIC en el proceso de compras de esta empresa. A continuación se muestran las respuestas a las preguntas relacionadas con el proceso de compras:

\section{En relación al proceso de negocio} ¿Cuál es el propósito de este proceso?

Adquirir los productos necesarios para ponerlos a la venta para satisfacer las necesidades de los clientes.

\section{¿Cuáles son las actividades involucradas?}

1.- Conocer cuáles son los productos faltantes en el inventario.

2.- Identificar quienes son los proveedores de esos productos.

3.- Elaborar la orden de compra para el proveedor.

4.- Enviar la orden de compra al proveedor.

5.- Mantener comunicación con el proveedor para dar seguimiento a la orden de compra.

6.- Surtir la orden de compra cuando llega la mercancía. 


\section{¿Quién realiza el proceso?}

El encargado del área de compras

¿Cuánto tiempo toma actualmente realizar el proceso y como se realiza?

Toma aproximadamente entre 30 y 50 minutos realizar una orden de compra. Se hace de manera manual en un formato impreso, después se escanea el documento y se envía el proveedor por correo electrónico.

\section{¿Cuándo se realiza el proceso?}

Cuando se detectan faltante de artículos para cubrir pedidos de los clientes. Esto suele suceder ya cuando al cliente se le dice que no se cuenta con el artículo solicitado después de realizar una búsqueda en el almacén, en consecuencia se pierde la venta para la empresa.

\section{¿Con que frecuencia se realiza el proceso?}

Es un proceso que se realiza diariamente en promedio para 10 proveedores

\section{¿Cuáles otras áreas se relacionan con el proceso?}

El proceso de compra tiene relación con:

a) El control de inventarios, ya que las compras realizadas afectan el nivel del inventario al registrarse las entradas de mercancía.

b) El departamento de pagos, ya que la compra puede ser contado o de crédito, afectando el estatus de las cuentas por pagar.

\section{¿Cómo se mejorará el aprovechamiento de las TIC en este proceso?}

Con la información capturada de los productos y los proveedores de mercancía, se puede utilizar para evitar la recaptura de los mismos datos y disminuir el tiempo para elaborar la orden de compra. El objetivo que se pretende alcanzar es conocer que artículos hay que comprar, a quien comprar y a qué precio comprar, así como aprovechar la conexión a internet para enviar automáticamente el documento de orden de compra al proveedor por correo electrónico. Con esto se aprovecha totalmente la aplicación del intercambio electrónico de datos entre las diferentes etapas que conforman el proceso de compras.

\section{Fase B: Adquisición y aplicación del conocimiento.}

En esta fase se siguieron las sugerencias del proveedor/asesor seleccionado para complementar algunos datos relacionados con el proceso de compra que involucra a los proveedores de mercancía, el catálogo de productos de la empresa y el proceso de elaboración de la orden de compra. 
En constante comunicación con el personal involucrado en esta fase se abordaron los siguientes puntos:

\section{Situación de la empresa en el uso del ERP actual}

La situación de la empresa acerca del uso del sistema informático actual se encuentra en un nivel bajo de aplicación en relación con el proceso de compras, ya que no se conoce en totalidad las funciones con las que cuenta el sistema para complementar este proceso.

\section{Información que presenta el sistema actual de la empresa}

Existen tres elementos que están relacionados con el proceso de compras: los proveedores, los productos y el proceso de elaboración de la orden de compra. A continuación se describe brevemente cada uno de estos elementos y su relación con el proceso de compras.

\section{Proveedores}

El catálogo de proveedores con los que cuenta la empresa es el contacto directo para realizar la petición los artículos. Se realizó un análisis de los tipos datos que se presentan para conocer cuáles son requeridos con otros procedimientos para aprovechar la conexión con las TIC que se requieran implementar. Si es necesario se deben definir nuevos tipos de datos, y especificar cuál es la función que tendría en las adecuaciones que se vayan a realizar para aprovechar el intercambio electrónico de datos en las diferentes actividades realizadas en el proceso, ya que con esto se ahorraría tiempo y se evitaría cometer errores en su captura.

\section{Productos}

El catálogo de productos es también un elemento muy importante, ya que se relaciona con la principal actividad de la empresa que es vender, pero también tiene relación relevante con el proceso de compras, ya que es la información principal que detalla en que consiste la compra que se está realizando y quienes son los proveedores. También se analizó cada uno de los tipos de datos que contiene, así como su función en el proceso de compras y otros procesos relacionados.

\section{Elaboración de orden de compra}

La orden de compra se define como el contenedor de la información relacionada con los proveedores y los artículos. En ella se detalla los artículos y las cantidades que son solicitadas, se puede llevar una relación de fechas cuando se realizan los pedidos así como los costos relacionados con la mercancía. Se analizó su estructura para saber cuáles tipos de datos son los adecuados para lograr que este documento tenga interacción entre las 
diferentes etapas del proceso de compra desde que se formule la petición hasta que se concluya con la adquisición de la mercancía y se efectué la entrada al inventario. De acuerdo a la adquisición del conocimiento de parte del proveedor/asesor, en esta fase se llegó a la conclusión que la aplicación del conocimiento adquirido, requirió incorporar algunos de datos en las tablas de información de los artículos, de los proveedores y de las órdenes de compra, con el fin de establecer una relación adecuada durante la ejecución del proceso de compras. En la figura 2 se presenta la relación entre estos datos.

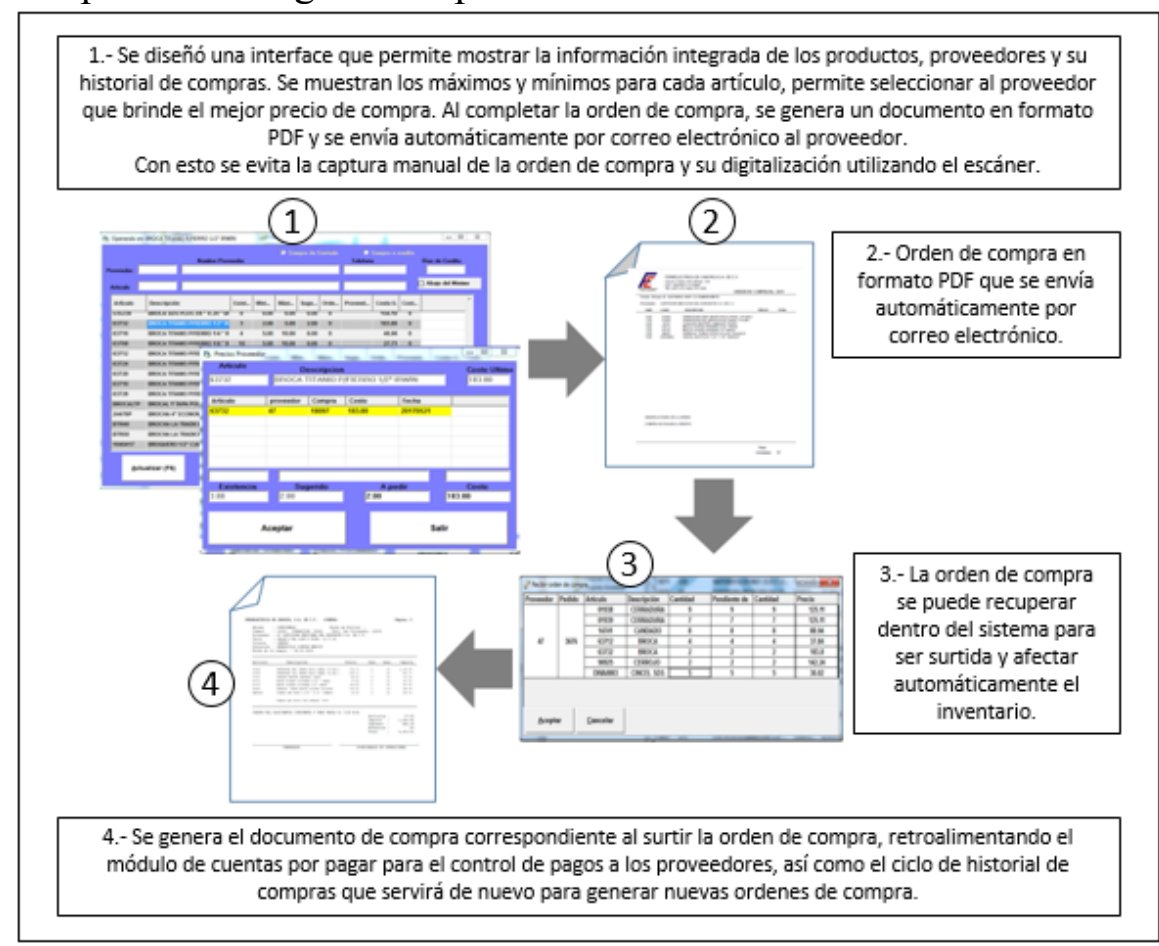

Figura 2.- Descripción de la solución implementada aplicando el modelo.

\section{Estado de evaluación 2.}

En este estado de evaluación la interrelación de los elementos del capital humano (conocimientos, destrezas, habilidades) permitió evaluar si la aplicación del conocimiento es favorable. En este punto se plantearon las siguientes preguntas relacionadas con la aplicación del conocimiento:

¿La aplicación de TIC está acorde al proceso de negocio analizado? ¿El proceso de negocio está apoyado por una estructura organizacional adecuada? ¿Las políticas de la empresa están soportadas adecuadamente por la estructura organizacional así como por los procesos y la tecnología aplicada? ¿El personal involucrado cuenta con los conocimientos y habilidades necesarias para la aplicación de TIC? En este caso, como las respuestas a estas preguntas por parte de la empresa fueron todas afirmativas, 
significa que se logró establecer en primera instancia un buen nivel acoplamiento entre los elementos del capital estructural. En caso de que este estado de evaluación no brindara resultados satisfactorios, se debe reforzar la fase B y analizar en consecuencia el estado de evaluación 1, lo cual podría llevar de regreso a reiniciar con la fase A, esto con el fin de avanzar a las etapas subsecuentes satisfactoriamente, o regresar entre fases y estados de evaluación anteriores como se considere necesario para lograr que se cumpla con el propósito de cada etapa del modelo.

\section{Fase C: Codificar y compartir el conocimiento.}

En esta fase se codificó el conocimiento, es decir, se documentó todo lo relacionado con el conocimiento aplicado de las TIC en el proceso de compras, así como las personas y las áreas de trabajo involucradas en dicho proceso. Se elaboró un manual de procedimientos describiendo los pasos necesarios para realizar el proceso de compra. El documento fue guardado en un archivo de procesador de texto en una carpeta específica para facilitar su almacenamiento y recuperación. Una vez codificado el conocimiento, la empresa ya cuenta con una fuente de conocimiento que puede consultar y actualizar de acuerdo a los cambios que se vayan presentando, logrando con ello mejores condiciones para compartir adecuadamente el conocimiento.

\section{Estado deseado}

Con la aplicación de este modelo, el estado deseado que se pretende es mejorar el nivel de aprovechamiento de las TIC en los procesos de negocio, y lograr al mismo tiempo la integración equilibrada de los tres factores del capital intelectual (capital humano, capital estructural y capital relacional). Con el fin de conocer el desempeño sobre la aplicación del modelo en el proceso de compras de la empresa, se dejó pasar un tiempo de 30 días trabajando con el nuevo proceso de compras. Se tomaron en cuenta 8 indicadores para elaborar un cuestionario y se aplicó a todas las personas relacionadas con este proceso. El cuestionario midió la percepción de las personas referente al mejoramiento del proceso de compras antes y después de aplicar el modelo. Se utilizó una escala de 1 a 5 (1 corresponde al nivel más bajo y 5 al nivel más alto de percepción). En la tabla 2 se muestran los promedios obtenidos del total de cuestionarios aplicados. En general las personas involucradas percibieron una mejora bastante significativa a la manera de cómo se realizaba el proceso de compra antes de aplicar el modelo. El puntaje acerca de la percepción del mejoramiento del proceso de compras aumentó positivamente en $179 \%$. Esto significa que todas las personas que participan en dicho proceso consideran que se ha mejorado sustancialmente el nivel de aprovechamiento de las TIC en el proceso de compras. 
Tabla 2.- Resultados de aplicación de modelo para mejorar el nivel de aprovechamiento de las TIC en el proceso de compras

\begin{tabular}{|c|c|c|c|}
\hline Indicadores a evaluar & $\begin{array}{c}\text { Promedio antes } \\
\text { del modelo }\end{array}$ & $\begin{array}{c}\text { Promedio } \\
\text { después } \\
\text { del modelo }\end{array}$ & $\begin{array}{c}\text { Diferencia } \\
\text { Antes-Después }\end{array}$ \\
\hline $\begin{array}{c}\text { Facilidad para integrar la } \\
\text { información } \\
\text { de artículos y proveedores }\end{array}$ & 2 & 4.5 & 2.5 \\
\hline $\begin{array}{c}\text { Facilidad para seleccionar } \\
\text { el mejor precio de compra }\end{array}$ & 1 & 5 & 4 \\
\hline $\begin{array}{c}\text { Tiempo de elaboración } \\
\text { de la orden de compra (OC) }\end{array}$ & 2 & 5 & 3 \\
\hline $\begin{array}{c}\text { Tiempo de revisión } \\
\text { y autorización de la OC } \\
\text { Tiempo para enviar } \\
\text { la orden de compra por e-mail }\end{array}$ & 2 & 5 & 3 \\
\hline $\begin{array}{c}\text { Tiempo de respuesta } \\
\text { por parte del proveedor }\end{array}$ & 2 & 4 & 4 \\
\hline $\begin{array}{c}\text { Facilidad para el surtido } \\
\text { de la OC }\end{array}$ & 1 & 5 & 3 \\
\hline $\begin{array}{c}\text { Nivel de aprovechamiento de } \\
\text { TIC }\end{array}$ & 2 & 5 & $\mathbf{3 . 0 7}$ \\
\hline en el proceso de compras & $\mathbf{1 . 7 1}$ & $\mathbf{4 . 7 9}$ & \\
\hline Promedio & & $59 \%$ & 3 \\
\hline Promedio \% & 2 & 5 & 3 \\
\hline
\end{tabular}

Fuente: Elaboración propia.

\section{Discusion}

De acuerdo a los resultados obtenidos de los indicadores establecidos se puede observar un mejoramiento considerable en el proceso de compras de la empresa (ver tabla 2). Con la aplicación del modelo se han realizado los procesos de la GC que se relacionan con la implementación de las TIC, y con los estados de evaluación correspondientes contenidos en el modelo se ha logrado mejorar el nivel de aprovechamiento en el proceso de negocio relacionado con las compras. Esto lleva a establecer que las TIC representan una gran oportunidad para que las PYMES mejoren su nivel de competitividad enfocándose en los procesos de negocio, pero su aplicación debe estar basada en medidas de eficiencia y productividad (Maldonado, García, Martínez, Aguilera y González, 2010). En consecuencia, la aplicación del modelo propuesto pone en práctica los procesos de la GC para avanzar sistemáticamente en la implementación de las TIC, mostrando evidencia de que las PYMES haciendo uso del conocimiento adecuado obtendrán mejores resultados en sus procesos de negocio logrando un buen equilibrio entre el capital humano, capital estructural y capital relacional, que aquellas PYMES que tengan TIC pero carecen del conocimiento para su implementación, y solo cuentan con un enfoque meramente de adquisición tecnológica y no basan su 
aplicación en la GC. Por tal razón debe quedar claro que el uso de las TIC no genera beneficios por el sólo hecho de adoptarlas, sino que sólo traerá beneficios a la empresa cuando se complemente con capacitación del capital humano, por ser el principal factor del cual depende la capacidad de absorción y la puesta en práctica del conocimiento (Rohrbeck, 2010).

\section{Conclusion y perspectivas futuras}

En consecuencia se puede observar que el aprendizaje continuo y la habilidad para desarrollar nuevas competencias en la aplicación de las TIC, las convierten en herramientas facilitadoras para las actividades empresariales. Aunque intervienen muchos factores en su implementación, el principal factor en que se debe sustentar es el capital humano, que en proporción a sus conocimientos, destrezas y habilidades hacen posible que surjan las ventajas de la incorporación de las TIC en las empresas en todos sus ámbitos como son gestión interna, comunicación con clientes y proveedores, sistemas de ventas, marketing, producción y recursos humanos.

En el escenario mexicano las PYMES representan una amplia área de oportunidad de mejoramiento en la implementación de la tecnología informática, por lo que se puede considerar para futuros trabajos aplicar este modelo en otros procesos de negocio en otras PYMES de diferente giro comercial y analizar su desempeño, con el fin de observar la adaptabilidad para implementar un enfoque sistémico en la aplicación de las TIC relacionándolas con la mayoría de los procesos de negocio de las empresas.

\section{References:}

1. Al-Sa'di, A. F., Abdallah, A. B., \& Dahiyat, S. E. (2017). The mediating role of product and process innovations on the relationship between knowledge management and operational performance in manufacturing companies in Jordan. Business Process Management Journal, 23(2), 349-376.

2. Alderete, M. V. (2013). Do information and communication technology access and innovation increase outsourcing in small and medium enterprises?.JISTEM-Journal of Information Systems and Technology Management, 10(2), 303-322.

3. Bradshaw, A., Cragg, P., \& Pulakanam, V. (2012, September). IS Consultants and SMEs: A Competence Perspective. In European Conference on Information Management and Evaluation (p. 25). Academic Conferences International Limited.

4. Cerchione, R., Esposito, E., \& Spadaro, M. R. (2015). The spread of knowledge management in SMEs: A scenario in evolution. Sustainability, 7(8), 10210-10232. 
5. Chinedu Eze, S., Duan, Y., \& Chen, H. (2014). Examining emerging ICT's adoption in SMEs from a dynamic process approach. Information Technology \& People, 27(1), 63-82.

6. Chong, A. Y.-L., Chan, F. T. S., \& Ooi, K.-B. (2012). Predicting consumer decisions to adopt mobile commerce: Cross country empirical examination between China and Malaysia. Decision Support Systems, 53(1), 34-43. doi:10.1016/j.dss.2011.12.001

7. Colin, M., Galindo, R., \& Hernández, O. (2016). Information and communication technologies, strategy and supply chain management in manufacturing SMEs of Aguascalientes, México. Annals of Data Science, 3(1), 71-88.

8. Dahiyat, S. E. (2015). An integrated model of knowledge acquisition and innovation: examining the mediation effects of knowledge integration and knowledge application. International Journal of Learning and Change, 8(2), 101-135.

9. Fey, C. F., \& Furu, P. (2008). Top management incentive compensation and knowledge sharing in multinational corporations. Strategic Management Journal, 29(12), 1301-1323.

10. Huaroto, C. A. (2012). "Efecto de la adopción de Internet en la productividad: Evidencia desde una muestra de microempresas en el Perú". ACORN-REDECOM, 17-18 de Mayo 2012, Valparaiso.

11. Maldonado, G. G., García, P. D., Martínez, S. M. C., Aguilera, E. L. y González, A. M. (2010) "La influencia de las TICs en el rendimiento de la PyME de Aguascalientes", Investigación y Ciencia de la Universidad Autónoma de Aguascalientes, No. 47, abril, pp. 57-65.

12. Miyazaki, S., Idota, H. y Miyoshi, H. (2012). Corporate productivity and the stages of ICT development. Information Technology Management, 13, 17-26.

13. Niedermann, F. (2015). Deep Business Optimization: concepts and architecture for an analytical business process optimization platform.

14. Park, M., Jang, Y., Lee, H. S., Ahn, C., \& Yoon, Y. S. (2013). Application of knowledge management technologies in Korean small and medium-sized construction companies. KSCE Journal of Civil Engineering, 17(1), 22.

15. Pedraza, N., Guerrero, M., \& Lavín, J. (2011). Alignment of EBusiness with SMEs' strategies in Northeast of Mexico. Journal of technology management \& innovation, 6(4), 205-224.

16. Radeschütz, S., Schwarz, H., \& Niedermann, F. (2015). Business impact analysis - a framework for a comprehensive analysis and optimization of business processes. Computer Science-Research and Development, 30(1), 69-86. 
17. Rantapuska, T., \& Sore, S. (2011). Progress of Commitment in Cooperative Software Acquisition. In Governance and Sustainability in Information Systems. Managing the Transfer and Diffusion of IT (pp. 224-235). Springer Berlin Heidelberg.

18. Rohrbeck, R. (2010) "Harnessing a network of experts for competitive advantage, technology scouting in the ICT industry", R\&D Management, Vol. 40, No. 2, United Kingdom, pp. 169-180.

19. Scupola, A. (2009). "SMEs' e-commerce adoption: Perspectives from Denmark and Australia". Journal of Enterprise Information Management, 22(1/2), 152-166.

20. Wang, S., \& Noe, R. A. (2010). Knowledge sharing: A review and directions for future research. Human Resource Management Review, 20(2), 115-131.

21. Wiig, K. M. (1997). Knowledge management: an introduction and perspective. Journal of knowledge Management, 1(1), 6-14.)

22. Wilkin, C. (2012). The role of IT governance practices in creating business value in SMEs. Journal of Organizational and End User Computing (JOEUC),24(2), 1-17. 